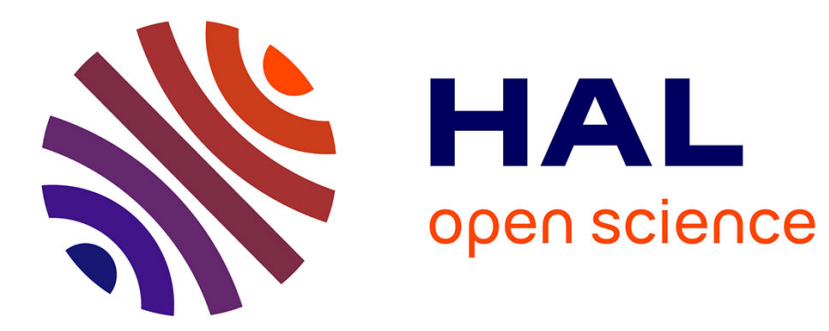

\title{
Assessing the Potential of IoT in Aerospace
}

Thirunavukkarasu Ramalingam, Benaroya Christophe, Fosso Wamba Samuel

\section{To cite this version:}

Thirunavukkarasu Ramalingam, Benaroya Christophe, Fosso Wamba Samuel. Assessing the Potential of IoT in Aerospace. 16th Conference on e-Business, e-Services and e-Society (I3E), Nov 2017, Delhi, India. pp.107-121, 10.1007/978-3-319-68557-1_11 . hal-01768513

\section{HAL Id: hal-01768513 \\ https://hal.inria.fr/hal-01768513}

Submitted on 17 Apr 2018

HAL is a multi-disciplinary open access archive for the deposit and dissemination of scientific research documents, whether they are published or not. The documents may come from teaching and research institutions in France or abroad, or from public or private research centers.
L'archive ouverte pluridisciplinaire HAL, est destinée au dépôt et à la diffusion de documents scientifiques de niveau recherche, publiés ou non, émanant des établissements d'enseignement et de recherche français ou étrangers, des laboratoires publics ou privés.

\section{(c)(1)}

Distributed under a Creative Commons Attribution| 4.0 International License 


\title{
Assessing the Potential of IoT in Aerospace
}

\author{
Thirunavukkarasu Ramalingam ${ }^{1}$, Benaroya Christophe ${ }^{2}$ and Fosso-Wamba Samuel ${ }^{3}$ \\ ${ }^{1}$ Indian Institute of Management, Bangalore, Karnataka, 560 076, India \\ 2, 3 Toulouse Business School, Toulouse, 31068 France \\ thirunavukkarasur15@iimb.ernet.in, c.benaroya@tbs-education.fr, \\ s.fosso-wamba@tbs-education.fr
}

\begin{abstract}
Internet of Things - Aerospace (IoTA): IoT in Aerospace sector? Is it really happening? This is what industry experts think while talking about Internet of Things in Aerospace! IoTA is the Ninth letter of Greek alphabet, which means 'extremely small amount'. Will it impact the nine letter 'AEROSPACE' industry? In the Aerospace \& Defense industry, split-second decisions can mean a difference between success and failure as there is longer product life cycle.

This exploratory research is targeted towards identifying the various IoT characteristics and its maturity level. A methodology was developed using House of Quality (HoQ) to identify the potential system which will be impacted by IoT in future. HoQ helped in identifying the positive and negative correlation between the IoT characteristics and its linkage with various aerospace systems. This investigative research can be used by aerospace system suppliers to make qualitative decisions before investing in IoT for their system/product enhancements.
\end{abstract}

Keywords: Internet of Things (IoT), Aerospace systems, IoT Characteristics, House of Quality (HoQ).

\section{$1 \quad$ Introduction}

Aerospace and Defense (A\&D) industry is poised for growth despite economic downturns. Increase in travel demand, development of new technologies and security threat for nations are fueling increase in aircraft production, defense budgets and the need for global supply chain [1]. Airbus global market forecast predicts that air traffic will grow at $4.5 \%$ annually and more than 30,000 aircrafts will be required over the next 20 years [2]. Aircraft manufacturers and operators are always in the lookout to improve the vehicle performance by providing more connected and smarter systems to achieve the Fuel Efficiency, Zero downtime and Route optimization.

The phrase Internet of Things (IoT) was first used in 1999 by Kevin Ashton. The concept of IoT is to connect the real-world objects with speech, vision, hearing, smell and touch, so inanimate things can perform jobs more accurately, responsively, collaboratively with learnings. IoT transformation is possible only when set of technologies are created that are broadly applicable to industry with relevant IoT characteristics maturity [3]. IoT characteristics defined as a set of capabilities encompasses in a system which can be increased or reduced based on design decisions and tradeoffs. 
Prior studies suggest that IoT may transform various industries and services including healthcare [4][5][6], construction [7], territories management [8], predictive maintenance [9] and manufacturing shop floors [10].

Nowadays digital transformation is the heart of business strategies and it begins with the executive mandate. There is a strong sense of urgency among executives as the threat of digitally enabled competitors and disruptive technologies remain high on the list of concerns. Internet of Things (IoT) is becoming more and more important in many industry sectors and domains. Digital Technology evolution is happening rapid fast, within no time this will impact every business. However, it is essentially a commitment by organizations to innovate which would add value to their customers.

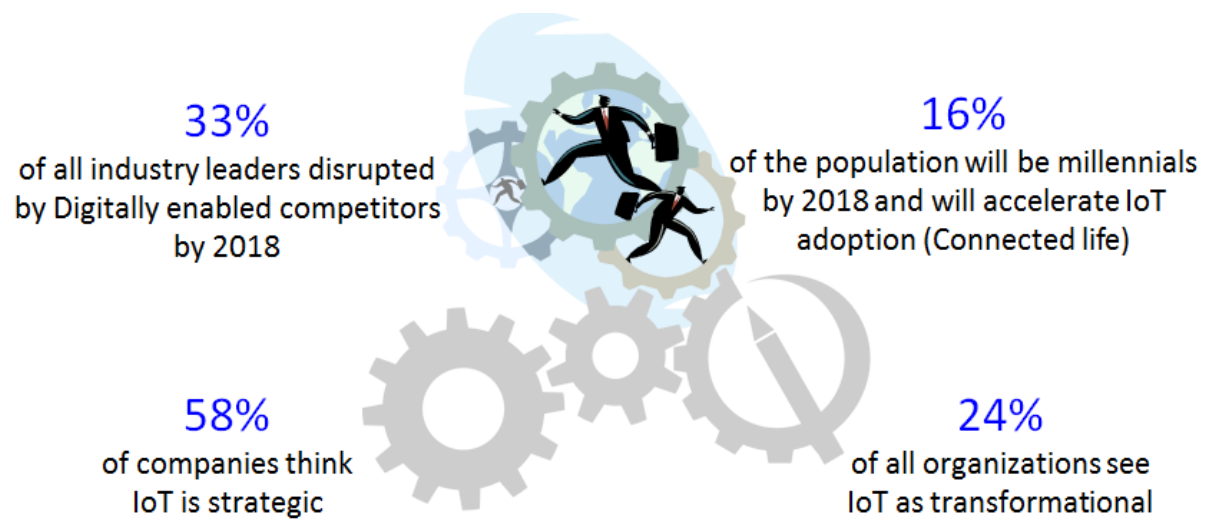

Fig. 1. Digital Transformation [11]

SAP research on digital transformation (see Fig. 1) shows that $33 \%$ of industry leaders will be disrupted in this way by 2018 and $58 \%$ of companies' think IoT is strategic [11]. Though the IoT evolution happened more than a decade ago, its impact on aerospace systems are limited due to IoT characteristics maturity, its adaptability and ease of implementation in safety critical aerospace systems.

\subsection{Objective of the paper}

Aerospace industries are currently in search of minimum viable systems/products and are investing heavily to develop IoT technologies which can yield long term value. IoT technologies are evolving faster than predicted which can change the product landscape in aerospace industry as well. However, there is no established approach to identify such systems/products. This investigative research paper can help aerospace system suppliers identify the IoT characteristics maturity. The methodology developed using HoQ will help them narrow down the system/product to focus.

According to the research paper [12] findings, it is indicated that the IoT research field is immature, with experimental methods dominating research outputs. No research paper covers IoT characteristics maturity and its impact on aerospace systems. Therefore, objectives of this exploratory research are as follows: 
$\checkmark \quad$ Identify and list the aerospace relevant IoT characteristics

$\checkmark$ Find out the IoT characteristics maturity

$\checkmark$ Connect the IoT characteristics with aerospace systems

\subsection{Author Definition of IoT \& IoTA}

Based on literature survey and its applicability to the context of this research, IoT is defined as "A system with in-built computing devices which can communicate with other such devices through secured network and take the decisions based on intelligence and can work on centralized and decentralized model".

Extending this definition to Internet of Things for Aerospace (IoTA), "IoTA is defined as aircraft systems with in-built computing devices which can communicate with other such devices through secured deterministic network and take the decisions based on swarm intelligence and works on centralized and decentralized model”.

\subsection{IoT Potential in Aerospace}

There are more than 20 IoT characteristics which can complement and add value in aerospace systems in many ways by reducing customer pain points such as flight cancellation, flight delays etc., Direct cost of air transportation delay is USD 32.9 billion which incurs a loss of USD 8.3 billion to airlines [13]. As per IATA report it is mentioned that USD 15 billion was spent on direct maintenance, with average maintenance cost of USD 295 million per airline and USD 1087 per flight hour [14]. Identification of potential systems and its relevant characteristics maturity is the key to develop and implement IoT products/systems in aerospace.

\section{Methodology}

Methodology followed in this project (see Fig. 2) was to first identify the various general IoT characteristics which are not relevant to any specific industry or domain. Each characteristics maturity was understood by conducting a targeted IoT audience market survey with varied experience level (Beginner, Intermediate \& Expert) across demographics (USA, Europe \& APAC). Two IoT experts were interviewed to validate the survey results and to confirm the IoT characteristics maturity. House of Quality (HoQ) tool was used to establish the correlation between the IoT characteristics and relations between the various aerospace systems. HoQ also helped to identify the relative weights between the characteristics and to identify potential IoT system.

\subsection{Level-1: IoT Characteristics Maturity}

Step-1: Identification of IoT characteristics using various published papers/articles Step-2: Definition of each IoT characteristics based on the literature survey Step-3: Online survey

- Develop interview questions with appropriate rating scale

- Circulate the online survey to targeted IoT audience across demographics

- Plot the survey results and analyze

Step-4: Survey result validation

- Conduct IoT experts' interview

- Co-relate the survey results and record findings 


\section{Limitations of Level-1}

- IoT characteristics definition and its understandability by survey audience

- Ratings by audience depends on their own perspective

- IoT experts' opinions based on their industry experience may/may not align with aerospace systems

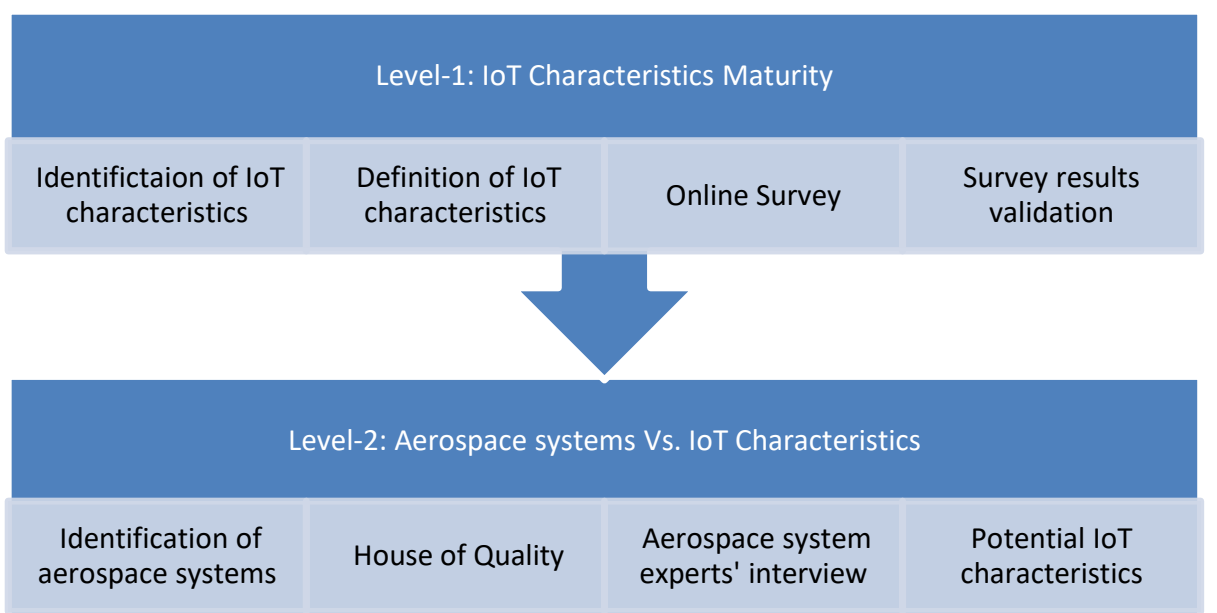

Fig. 2. Step by Step methodology for IoTA

\subsection{Level-2: Aerospace systems Vs. IoT characteristics}

Step-1: Identification of top level aerospace systems

Step-2: Develop House of Quality

Step-3: Aerospace system experts' interview

○ Identify system level experts'

- Explain the context of the discussion and conduct focused interview

- Use House of Quality (HoQ) to establish the linkages between the aerospace systems and IoT characteristics

Step-4: Potential IoT characteristics

- Establish the correlation between the IoT characteristics

In this project, Authors have rated each characteristic to establish the correlation (Triangular shaped Roof) based on insight, gained through the literature survey.

- Identification of potential IoT characteristics for each aerospace system

\section{Limitations of level-2}

- Experts' idea on future aerospace system using IoT was limited

- Definition of IoT characteristics and its explanations to the system level experts'

- IoT experts rating and its validation of results 


\section{IoT Characteristics}

Table 1 shown below lists the IoT characteristics that are fundamental and emerging new characteristics mentioned/referred in published articles. Online survey participants suggested characteristics are also listed.

Table 1. IoT characteristics

\begin{tabular}{|c|c|}
\hline Fundamental IoT characteristics[15] & Mentioned/referred in published articles \\
\hline $\begin{array}{ll}\text { - } & \text { Interconnectivity } \\
\text { - } & \text { Things-related services } \\
\text { - } & \text { Heterogeneity } \\
\text { - } & \text { Dynamic changes } \\
\text { - } & \text { Enormous scale } \\
\text { - } & \text { Safety } \\
\text { - } & \text { Connectivity }\end{array}$ & $\begin{array}{ll}\text { - } & \text { Intelligence } \\
\text { - } & \text { Sensing / Sensor } \\
\text { - } & \text { Expressing } \\
\text { - } & \text { Energy } \\
\text { - } & \text { Computing/ Processors } \\
\text { - } & \text { Quality \& Reliability } \\
\text { - } & \text { Cost effectiveness } \\
\text { - } & \text { Consumption } \\
\text { - } & \text { Conversion } \\
\text { - } & \text { Centralization } \\
\text { - } & \text { Cognition } \\
\text { - } & \text { Configuration } \\
\text { - } & \text { Coordination } \\
\text { - } & \text { Deterministic } \\
\text { - } & \text { Mobility } \\
\text { - } & \text { Security }\end{array}$ \\
\hline \multicolumn{2}{|l|}{ Suggested by online survey participants } \\
\hline $\begin{array}{ll}\text { - } & \text { Miniaturization \& Composa } \\
\text { - } & \text { Standards and Protocols } \\
\end{array}$ & \\
\hline
\end{tabular}

Though 25 IoT characteristics were identified, all of them may not contribute to aerospace systems. Definition of 11 IoT characteristics are explained in section 3.1 that have scored a relative weight above 4 in HoQ and are favorable for IoT systems/ products in aerospace industry.

\subsection{Definition of IoT characteristics}

Definitions of potential IoT characteristics for aerospace systems development are listed below:

Safety: IoT can yield lot of benefits, however safety should be the prime concern. Design for safety is the key aspect to be considered both by creators and recipients of the IoT. This includes the safety of personal data and physical well-being [15].

Connectivity: It enables network compatibility and accessibility. Compatibility provides the common ability to consume and produce data while accessibility is getting on a network. Connectivity in the IoT can very soon get rid of the Wi-Fi module [15]. 
Intelligence: Product experiences are made smarter with the help of software and hardware algorithms and its computing power provides the "intelligent spark" [16].

Expressing: Expressing provides a way to create products that interact intelligently with people and real world, not just rendering beautiful user interface [16].

Energy-Efficiency: Energy harvesting, power efficiency and charging infrastructure are the necessary parts of a power intelligent ecosystem that we must design. Today, it is woefully inadequate and lacks the focus in many product teams [16].

Computing/ Processors: Certain degree of computing power is needed for all the devices connected in the network so that IoT devices can relay and transmit gathered data. Like any other computing device, this will require a processor as well [17].

Quality \& Reliability: Devices in the IoT may be operating in extreme weather conditions and tough environments. As the IoT devices might be exposed to such an environment it is important that they are made with the highest quality and reliability [17]. Cognition: This is not the same as plain old data conversion it is more of an analytical process where we apply context to the data in hand. Cognition makes sure that we get the right perception of the data [18].

Deterministic: Time Sensitive Networking (TSN) features are currently being developed by The IEEE standards organization by including standard 802.1 and 802.3. To allow Ethernet to be deployed in mission critical applications, it is necessary to add specific features including time synchronization, scheduled traffic, ingress policing and seamless redundancy. This is to ensure that specific data traffic can flow, on time and throughout the entire network topology [16].

Security: This is concerned with safeguarding IoT connected devices and networks. Security is a wide concept which covers everything from authenticity, authority, integrity and confidentiality. Security paradigm will scale up when IoT would need securing the endpoints, the networks and the data moving across the network [19].

Standards \& Protocols: A standard document has all rules and procedures developed by a regulatory party and agreed upon to be followed by many parties. A protocol is a particular set of rules that enables conversation between two computers to convey a specific set of information. Such well known communication technologies are Wi-Fi, Bluetooth, ZigBee and 2G/3G/4G cellular [20].

\subsection{IoT Characteristics Maturity}

More than 20 IoT characteristics were identified by going through various whitepapers, technical papers and published articles on Internet of Things. As these characteristics have evolved over several years, it is not easy to measure its maturity from those data sources. Hence authors have decided to conduct market research using targeted IoT audience and validate the survey results by interviewing IoT experts'.

A survey questionnaire is formulated for the

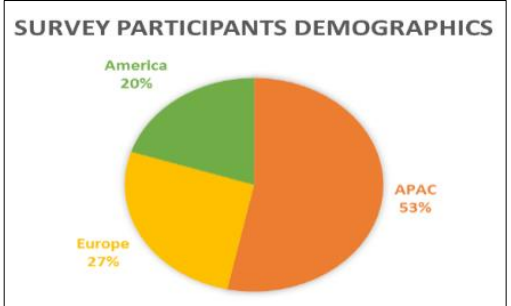

Fig. 3. Survey Demographics target audience with explanation of the identified IoT characteristics and a four-point scale rating to assess the maturity (i.e. New, Improving, Maturing \& Aging). The target 
audience/survey participants were carefully chosen (with IoT experience of 2 years at least) across the globe as there are not many IoT industry experts. Fig. 3 show the percentage of participants across demographics.

About 30 responses were received from across the globe and its results are shown in Fig. 4. More than 10 characteristics have scored above 50\% rating on 'Improving' scale (see Fig. 4). Two characteristics (connectivity and computing \& processor) have scored above $30 \%$ on 'Mature' scale (see Fig. 5). Online survey respondents helped in identifying two more characteristics - Miniaturization \& Composability and Standards \& Protocols. These two characteristics were later included for rating during the IoT experts' interview.

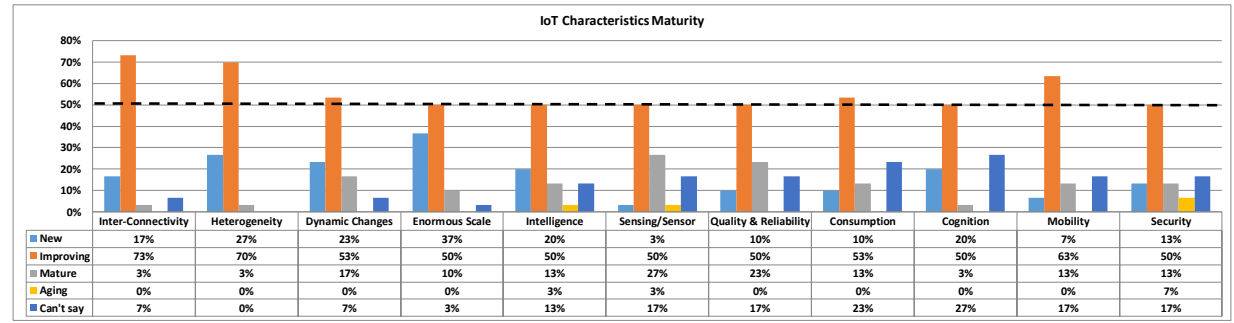

Fig. 4. IoT Characteristics $\geq 50 \%$ in 'Improving' scale

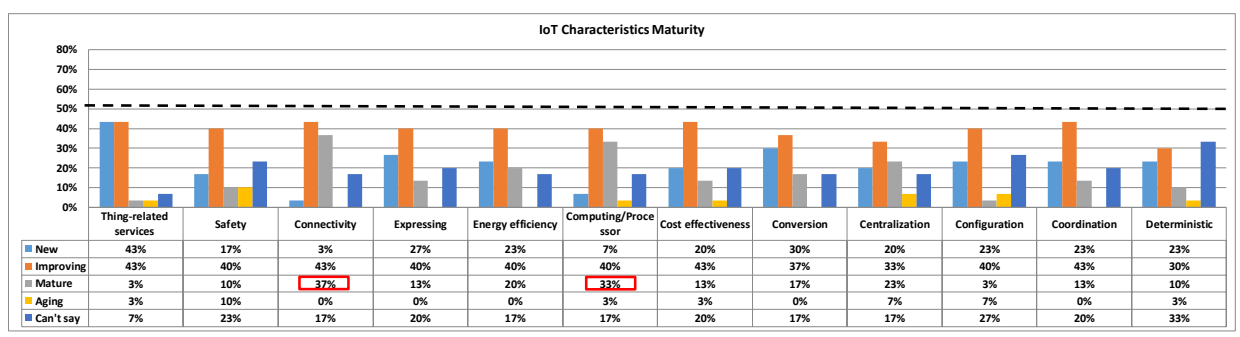

Fig. 5. IoT Characteristics $<50 \%$ in 'Improving' scale

\subsection{Survey Result Validation}

To validate survey results, two industry experts were interviewed by the author. Expert1 is a Senior Director of leading USA multinational company pioneer in Industrial Internet of Things (IIoT). Expert-2 is a Product Marketing Manager in USA aerospace company which is actively pursuing the IoT journey in aerospace industry.

There are differences between survey results and experts' ratings due to the following reasons.

- Personal interviews provide more subjective data than surveys. It was very evident from the two sets of charts that depicted the similarities and differences in perspectives of IoT experts online survey participants.

- Experts were asked to rate in the scale of 1-5 to reduce the ambiguity. Results of interview might not be statistically reliable, but closer ratings were grouped together. 
- Experts' interviews yielded valuable insights into customer attitudes and unfolded issues related to new IoT products development in their respective industry.

Similarities in ratings by experts' and survey (see Fig. 6) were observed for the characteristics such as Inter-connectivity, Dynamic changes, Things related services, Sensing/ Sensor, Expressing, Quality \& Reliability, Cost-effectiveness, Consumption, Conversion, Centralization, Cognition and Standards \& Protocols.

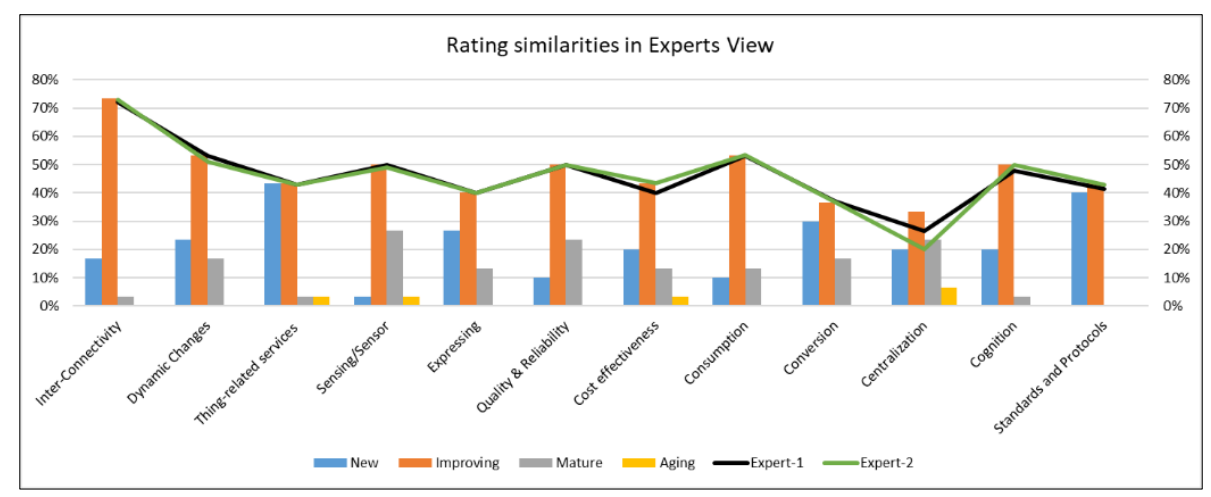

Fig. 6. Similarities in survey result \& Experts' view

Expert-1 ratings on maturity have largely varied from survey results for the characteristics such as Heterogeneity, Safety, Connectivity, Energy efficiency, Computing/processor and Security. Whereas, IoT Characteristics such as Enormous scale, Intelligence, Configuration, Coordination, Deterministic, Mobility and Standardization \& Composability have closely aligned rating on maturity levels with the survey results (see Fig. 7).

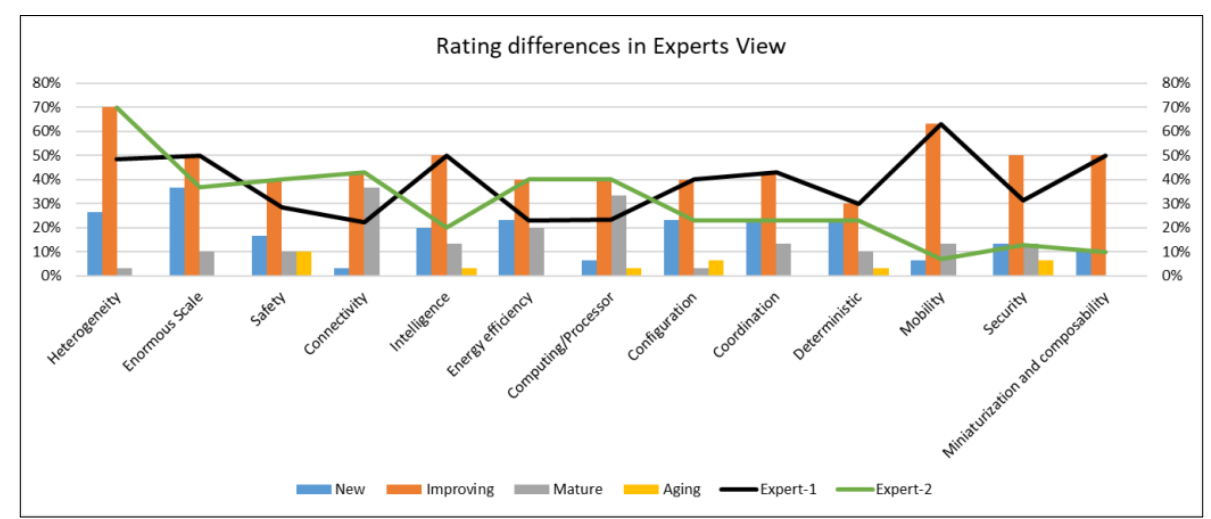

Fig. 7. Differences in survey result \& Experts' view

As per Expert-2, Enormous scale, Intelligence, Configuration, Coordination, Deterministic, Mobility, Security and Standardization \& Composability have different maturity ratings compared to the survey results. IoT characteristics such as Heterogeneity, 
Safety, Connectivity, Energy efficiency and Computing/processor rating of Expert-2 on maturity ratings have aligned with survey results (see Fig. 7).

IoT characteristics 'Mobility' has received completely varied Maturity ratings from expert-1 and expert-2 (See Fig. 7). Industrial IoT expert has rated 'Mobility' on 'Improving' scale whereas Aerospace IoT expert has rated the same as 'New'. This may be due to their different industries of work and limited use of 'Mobility' characteristics except for its application in Fight Entertainment (IFE) and ground handling equipment, in aerospace domain. It means that this particular characteristic 'Mobility' needs lot of improvement before adopting it to the aerospace industry. It is rightly pointed out by expert-1 that there is a need for 'Mobility' characteristics and lot of development/improvement efforts are happening in that area. According to the experts', more number of characteristics have aligned with Industrial IoT than with aerospace IoT industry, which makes it clear that Industrial IoT products are yielding more value than aerospace IoT products. It shows that safety critical aerospace industry has just started off the IoT journey.

\section{Aircraft Avionics, Systems and Equipment}

There are many aircraft systems and sub systems behind every successful flight. To establish the relationship between the IoT Characteristics and aircraft systems, the authors have decided to use research areas listed (see Table 2) in European Aeronautics Science Network (EASN) [21].

Table 2. List of Aircraft Avionics, Systems and Equipment

- Avionics

- Cockpit Systems, Visualization \& Display Systems

- Navigation / Flight Management / Auto-land

- Warning System

- Electronics \& Microelectronics for on-board systems

- Sensors integration

- Flight Data/Flight Recording

- Communications Systems

- Identification of Aircraft by ATM (Air traffic Management)

- Avionics Integration

- Optics - Optronic - Lasers - Image processing and data fusion
- Electronic Library System

- Aircraft health and usage monitoring system

- Smart maintenance systems

- Lighting systems

- Aircraft Security

- Electrical Power Generation \& Distribution

- Pneumatic systems

- Hydraulic power generation \& distribution

- Passenger and freight systems

- Environmental control System

- Water and waste systems

- Fuel systems

- Landing gear and braking systems

- Fire protection systems 


\section{$5 \quad$ Integrating All}

'House of Quality' (HoQ), the basic design tool for the management approach known as Quality Function Deployment (QFD), originated in 1972 at Mitsubishi's Kobe shipyard site. Toyota and its suppliers developed it in numerous ways. HoQ is a kind of conceptual map that provides the means for inter-functional planning and communication. People with different industrial problems and responsibilities can thrash out design priorities while referring to patterns of evidence on the house's grid [22].

HoQ is one of the matrices of an iterative process called QFD. It is the nerve center that drives the entire QFD process which is most recognized and widely used tool for new product design. It translates customer requirements, based on market research data, into an appropriate number of engineering targets to be met by a new product design. It is performed by a multidisciplinary business critical team [23].

Customer attributes for aircraft systems are listed on the left of the matrix (Horizontal). Meaningful, measurable and global IoT characteristics are listed in the top column of HoQ. Relationship matrix (Central portion) between aircraft systems and IoT characteristics are established (scale of strong, medium and weak relationships) using the discussion outcome with aerospace system experts' (17 interviews with experience levels in the range of 7-30 years) who are working for Tier-1 aircraft system companies. End user views from Pilots, Maintenance Engineers \& Airlines executives ( 3 interviews with experience levels in the range of 10-20 years) are also considered. Five system relationships are not evaluated due to lack of time with system experts' and are marked in red color.

Determined Co-relationship matrix (Triangular shaped Roof) with potential positive and negative interactions between IoT characteristics. Too many positive interactions imply possible redundancy in aircraft systems IoT characteristics. Negative interactions suggest to consider the trade-offs in establishing target values for aircraft systems concepts or technology (see Fig.8).

As per the survey results and experts' interview, most of the characteristics are difficult and risky to implement in aerospace systems as the maturity levels are still new and improving. 15 Out of 25 characteristics have scored relative weight $\geq 4$ (Dynamic changes, Safety, Connectivity, Intelligence, Expressing, Energy efficiency, Computing and processor, Quality and Reliability, Cognition, Configuration, Deterministic, Security and Standards \& Protocols). These characteristics can potentially impact aerospace systems design and its perceived value in future.

\subsection{Assumptions}

- Survey participants' knowledge level on IoT characteristics maturity is moderate

- Aerospace systems experts understand the basics of IoT and its impact

- Selection of Aerospace system is based on the research interest 


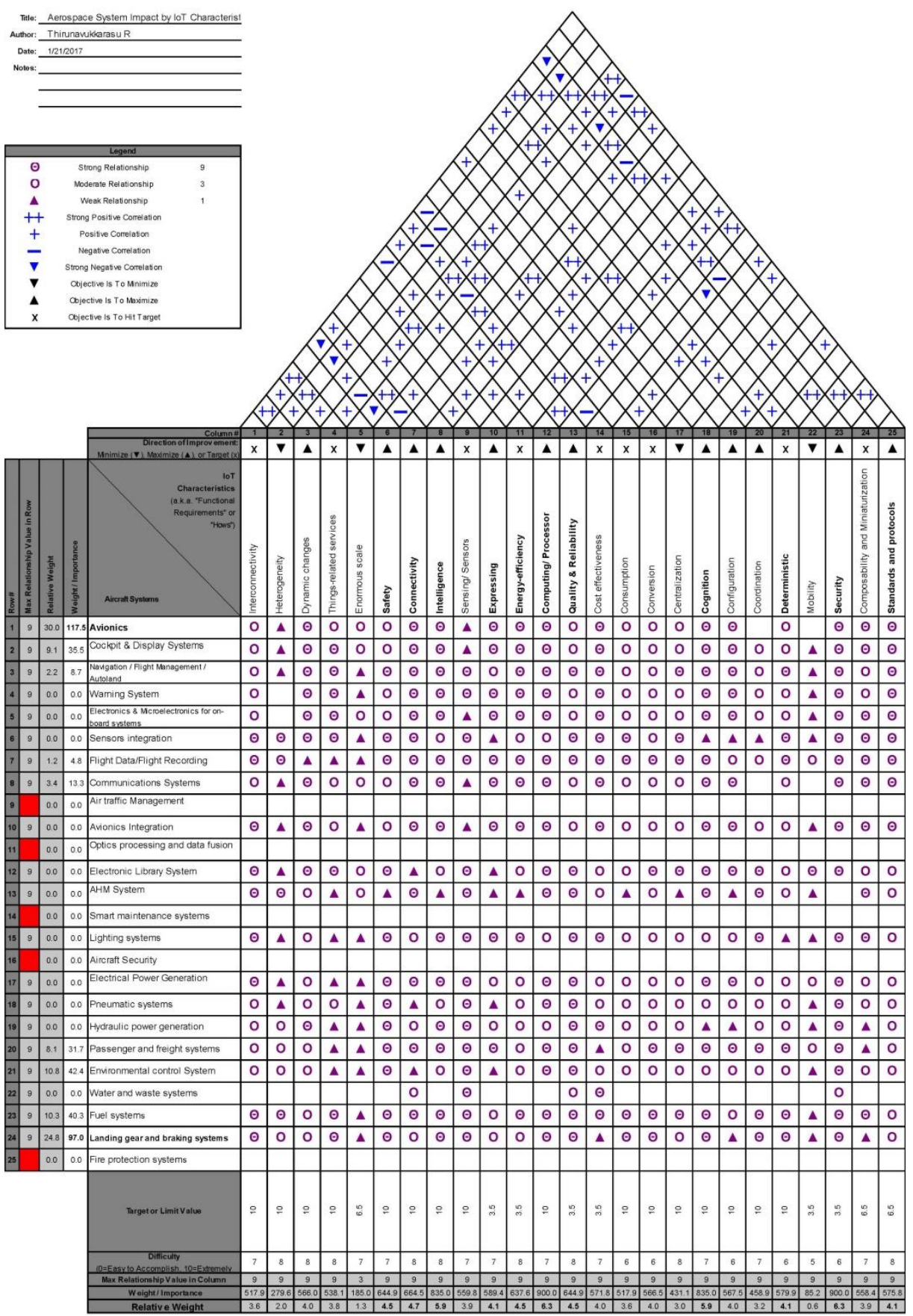

Fig. 8. HoQ IoTA 


\subsection{Limitations}

- Use of HoQ alone to establish the relationship between the IoT characteristics

- Perspective of IoT impact on aerospace systems can vary among experts

- IoT characteristics maturity are evolving every day

- Risk factor of using weak relationships in HoQ rows or columns

○ Too many relationships between the characteristics

\subsection{Results of HoQ}

HoQ reveals the relative weight of the IoT characteristics. Four characteristics have scored more than 5 (Intelligence, Computing/Processor, Cognition, and Security). It would encourage companies to invest in developing technologies which can mature on these characteristics. Characteristics such as Intelligence \& Computing/Processor have evolved over a period of time and have benefited other industries. It's time for aerospace industry to develop IoT products using the available technologies. Cognition remains as a challenge for IoT products as data is still being interpreted and perceived. Currently less than $1 \%$ of data is used for decision making. Security is another challenge for IoT in aerospace industry as the standards and protocols are still evolving and yet to be established.

\section{$6 \quad$ Recommendations}

By collating the ratings on IoT characteristics maturity on aerospace systems provided by IoT and Aerospace systems experts', the weightage/importance are evaluated. Avionics and Landing systems have scored high among the aerospace systems. Also, these systems have strong relationship with $50 \%$ of the IoT characteristics, which are on improving scale. Aircraft system suppliers should look out for the opportunities in \& out of their domain to develop IoT systems/products which can yield long term value to their organizations. Based on this exploratory research, paper authors recommend the following:

$\checkmark \quad$ Methodology can help in identifying the potential aerospace system for implementing IoT

$\checkmark$ HoQ will help to develop relationship between various IoT characteristics and aircraft system

$\checkmark$ When the maturity scale for an IoT characteristic increases, the ease of its implementation in aircraft system also increases

HoQ also helps in establishing the relationship between the characteristics and aircraft systems. Aircraft system development companies are likely to focus on co-relations between the IoT characteristics shown in Triangular shaped roof (see Fig.8). 


\section{Conclusions}

This research project has established the relationship between IoT Characteristics maturity and its impact on Aerospace systems. HoQ has helped in identifying the potential aerospace systems where IoT can be implemented. The methodology referred in this paper can help aerospace systems companies to understand the IoT characteristics and its related impact on aerospace systems. This paper has identified IoT characteristics which are relevant to aerospace industry. IoT characteristics maturity was determined by an online survey with targeted audience and by interviewing IoT experts. Finally, the IoT Characteristics were connected, relatively weighed and its relationship with system was established by interviewing aerospace system experts'. As per research findings, characteristics such as Intelligence, Computing/Processor, Cognition and Security have scored relative weight $>5$. This means that these characteristics are key for implementing IoT in aerospace systems. Aerospace companies should analyze the maturity of these characteristics before considering to invest in developing IoT products.

Future scope of research is to develop a robust methodology to find out the IoT characteristics maturity with increased number of target audience. Aerospace systems experts' interviews can be strengthened further by framing the questionnaire targeting their proficiency level and its relevance to IoT. Research can be extended further on the lines of identifying the impact of IoT elements like sensing, communicating etc., on aerospace systems. HoQ shall be developed for each aerospace system with IoT elements which can help companies move forward in developing IoT enabled aerospace systems.

\section{References}

1. Bénaroya, Ch. \& Malaval, Ph., (2013), Aerospace Marketing Management, Berlin, Springer.

2. Global Market Forecast 2016-2035 - Airbus

3. Amy J.C. Trappeya, Charles V. Trappeyb, Usharani Hareesh Govindarajana, Allen C. Chuanga, John J. Suna "A Review of essential standards and patent landscapes for the IoT: Key enabler for Industry 4.0" Advanced Engineering Informatics, 2016 pp. 3

4. Fosso Wamba, S. 2012. "Rfid-Enabled Healthcare Applications, Issues and Benefits: An Archival Analysis (1997-2011)," Journal of Medical Systems), pp. 1-6.

5. Rahmani, A.M., Gia, T.N., Negash, B., Anzanpour, A., Azimi, I., Jiang, M., and Liljeberg, P. 2017. "Exploiting Smart E-Health Gateways at the Edge of Healthcare Internet-of-Things: A Fog Computing Approach," Future Generation Computer Systems).

6. Wamba, S.F., and Ngai, E.W.T. 2013. "Internet of Things in Healthcare: The Case of RfidEnabled Asset Management," International Journal of Biomedical Engineering and Technology (11:3), pp. 318-335.

7. Zhong, R.Y., Peng, Y., Xue, F., Fang, J., Zou, W., Luo, H., Thomas Ng, S., Lu, W., Shen, G.Q.P., and Huang, G.Q. 2017. "Prefabricated Construction Enabled by the Internet-ofThings," Automation in Construction (76), 4//, pp. 59-70.

8. D'Angelo, G., Ferretti, S., and Ghini, V. 2017. "Multi-Level Simulation of Internet of Things on Smart Territories," Simulation Modelling Practice and Theory (73), 4//, pp. 3-21. 
9. Civerchia, F., Bocchino, S., Salvadori, C., Rossi, E., Maggiani, L., and Petracca, M. 2017. "Industrial Internet of Things Monitoring Solution for Advanced Predictive Maintenance Applications," Journal of Industrial Information Integration.

10. Tan, Y.S., Ng, Y.T., and Low, J.S.C. 2017. "Internet-of-Things Enabled Real-Time Monitoring of Energy Efficiency on Manufacturing Shop Floors," Procedia CIRP (61), //, pp. 376-381.

11. Lynne Dunbrack, Simon Ellis, Leslie Hand, Kimberly Knickle, Vernon Turner, 2016 White Paper "IoT and Digital Transformation: A Tale of Four Industries" Sponsored by: SAP

12. Marta Vos, 2015 "Maturity of the Internet of Things Research Field: Or Why Choose Rigorous Keywords" Australasian Conference on Information Systems, Adelaide, //, pp-1

13. Michael Ball, Cynthia Barnhart, Martin Dresner, Mark Hansen, Kevin Neels, Amedeo Odoni, Everett Peterson, Lance Sherry, Antonio Trani, Bo Zou, 2010, Research Report published by The National Centre of Excellence for Aviation Operations Research titled "Total Delay Impact - A Comprehensive Assessment of the Costs and Impacts of Flight Delay in the United States" // pp-14

14. An Exclusive Benchmark Analysis (FY2014 data) by Maintenance Cost Task Force published by IATA, https://www.iata.org/whatwedo/workgroups/Documents/MCTF/AMCExec-Comment-FY14.pdf

15. Keyur K Patel, Sunil M Patel "Internet of Things-IOT: Definition, Characteristics, Architecture, Enabling Technologies, Application \& Future Challenges” International Journal of Engineering Science and Computing, May 2016 // pp-6123.

16. http://www.electronicsweekly.com/news/ethernet-goes-deterministic-for-iot-2016-02/

17. https://www.webchoiceonline.com.au/blog/?post=63

18. http://www.forbes.com/sites/adrianbridgwater/2016/01/12/the-7-cs-of-the-internet-ofthings/\#124784ce59f4

19. Antonio J. Jara, Latif Ladid, Antonio Skarmeta, "The Internet of Everything through IPv6: An Analysis of Challenges, Solutions and Opportunities" Journal of Wireless Mobile Networks, Ubiquitous Computing, and Dependable Applications, volume: 4, number: 3

20. https://www.rs-online.com/designspark/eleven-internet-of-things-iot-protocols-you-needto-know-about

21. https://easn.net/ and https://easn.net/research-technology-areas/4/\#60

22. https://hbr.org/1988/05/the-house-of-quality

23. Praveen Shrivastava "House of Quality: An Effective Approach to Achieve Customer Satisfaction \& Business Growth in Industries" International Journal of Science and Research (IJSR) Volume 5 Issue 9, September 2016. // pp-1365. 